\title{
Quality of Life in Patients With Severe Skin Reactions in Course of First-Generation Epidermal Growth Factor Receptor Inhibitors Monoclonal Antibodies (Our Experience With Cetuximab)
}

\author{
Rossella De Luca ${ }^{\mathrm{a}}$, Gianluca Lo $\mathrm{Coco}^{\mathrm{b}}$, Raffaele Addeo ${ }^{\mathrm{c}, \mathrm{e}}$, Silvia Ileana Sara Fattoruso ${ }^{\mathrm{c}}$, \\ Annunziata Auriemma ${ }^{c}$, Roberta Pacia ${ }^{a}$ Ornella Mistretta ${ }^{a}$, Maria Stella Epifanio ${ }^{b}$, \\ Angela Salvato $^{c}$, Alberto D'Agostino ${ }^{\text {, }}$, Giuseppe Cicero ${ }^{\text {a, e }}$
}

\begin{abstract}
Background: Metastatic colorectal cancer (mCRC) with wild type expression of RAS and RAF genes can be treated with anti-epidermal growth factor receptor (EGFR) monoclonal antibodies, such as cetuximab, in combination with chemotherapy. Skin toxicity represents the most serious and frequent side effect in these patients. Skin manifestations occur in approximately $80 \%$ of patients. In this study, we investigated the consequences on body image and quality of life (QoL) of patients with severe skin toxicity.
\end{abstract}

Methods: One hundred patients were enrolled with mCRC. All patients signed informed consent and completed questionnaires to assess QoL and body discomfort. Toxicity was assessed on Common Terminology Criteria for Adverse Events (CTCAEs).

Results: The greatest impact on QoL was represented by difficulties in managing skin rash-related side effects. Data showed a significant impact in psychological sphere and social relationships.

Conclusions: Skin side effects, particularly rash, influence QoL and social relationships, compromising therapeutic compliance.

Manuscript submitted April 15, 2021, accepted May 20, 2021

Published online July 10, 2021

${ }^{a}$ Department of Surgical, Oncological and Oral Sciences, Section of Medical Oncology, University of Palermo, Palermo, Italy

${ }^{b}$ Department of Psychology and Educational Sciences, University of Palermo, Palermo, Italy

'Oncology Operative Unit, Hospital of Frattamaggiore (NA), ASLNA2NORD, Italy

'Surgery Unit and Trauma Center, AORN Sant'Anna e San Sebastiano, Caserta, Italy

${ }^{e}$ Corresponding Authors: Raffaele Addeo, U.O.C. Oncologia, "San Giovanni di Dio" Hospital, ASLNA2NORD, Via Pirozzi 62, 80027 Frattamaggiore (NA), Italy. Email: raffaeleaddeo19@gmail.com; Giuseppe Cicero, Department of Surgical, Oncological and Oral Sciences, University of Palermo, Via del Vespro 129, 90127 Palermo, Italy. Email: giuseppe.cicero@unipa.it

doi: https://doi.org/10.14740/wjon1381
Keywords: Quality of life; Cetuximab; Chemotherapy; Metastatic colorectal carcinoma; Metastases; Skin rash; Psychological reactions

\section{Introduction}

Metastatic colorectal cancer (mCRC) is the second cause of cancer death in Europe and the third most common cause of cancer mortality in USA. About $35 \%$ of patients with mCRC have clinically detectable liver metastases at diagnosis and approximately $45 \%$ develop metastases during disease [1]. The 5 -fluorouracil plus leucovorin became the standard of care of this disease, with median overall survival (OS) of 8 - 9 months. The addition of oxaliplatin and irinotecan, with FOLFOXFOLFIRI regimens, gained $18-20$ months [2] and the introduction of new drugs into clinical practice has progressively improved survival in advanced disease, bringing median survival up to 24 months, and in some cases the drugs have allowed to convert the disease from unresectable to resectable [2-4].

Furthermore, biological agents as epidermal growth factor receptor (EGFR) or vascular endothelial growth factor receptor (VEGFR) inhibitors seem to offer further therapeutic options [5]. The monoclonal antibody cetuximab $\left(\operatorname{Erbitux}^{\circledR}\right)$ is the EGFR inhibitor most studied and approved by FDA for mCRC treatment with EGFR expression and without RAS gene mutations (Ras wild type) approved in first and later lines of treatment $[6,7]$.

Cetuximab has an important therapeutic effect on metastatic disease, and improves survival curves and overall response rate. In about $80 \%$ of patients, cetuximab causes disfiguring skin toxicity, mainly in the face and neck. The skin lesions begin as a diffuse facial erythema also in the neck, flaking, dry skin with diffuse and itchy folliculitis similar to acne, which evolves into pustules with formation of strongly adherent yellowish crusts [7-9].

The most commonly reported adverse event is acneiform rash, typically mild or severe in up to $18 \%$ of patients $[10$, 
11]. Patients often experiment edema and erythema during first week of therapy following by papules, pustules and crusting. Paronychia and fissures are latter events.

The papules and pustules are often accompanied by telangiectasias, with an image similar to that of rosacea. These acneiform eruptions are often associated with microbial infections. In persistent lesions, the bacterial colonization with Staphylococcus aureus and/or detection of herpes simplex type I is not uncommon. In $15 \%$ of cases, skin manifestations can be so severe as to cause skin necrosis, nail changes (paronychia) and inflammation with eyes redness, tearing and sensitivity to light with blurred vision [12].

Eczematization and bacterial overlap are complications that can occur in areas less resistant to infections; in these cases, the choice treatment is based on low-dose topical corticosteroids in combination with antibiotics.

The main pharmacological treatment is the oral or topical application of tetracycline family, such as doxycycline or minocycline, antibiotics or corticosteroid alone or in combination, accompanied by sun creams with protection factors [13].

Painful fissures were observed in fingers and toes, alterations of the plate nail and paronychia, after 2 months of treatment, as well as pyogenic granulomas $[14,15]$. Most skin reactions develop within first or third week of therapy. However, the presence and severity of rash is associated with better clinical efficacy in patients being treated with EGFR inhibitors [16].

Furthermore, the reaction to treatment varies from individual to individual and, depending on treatment type performed, moreover, not all patients undergoing therapy show side effects [17].

In severe cases that clearly disfigure patients' faces, treatment discontinuation is necessary.

Skin lesions from cetuximab often disfigure patient's face and physical appearance with a serious psychological impact that leads patient to withdraw the social, a similar impact such as alopecia or mutilation of surgical procedures such as mastectomy or colectomy $[18,19]$. A multidisciplinary approach, with a psycho-oncologist in team, is the best way to properly manage the patient with mCRC [20]. At least two-thirds of patients with mCRC suffer side effects associated with chemotherapy that cause distress, anxiety and depression. These side effects are a determining component in the experience of the disease that significantly compromise quality of life (QoL). In fact, most patients fear, not so much by the disease but by physical and psychological consequences to treatment [21]. There is a negative modification of image and self-concept, a fall in self-esteem, lifestyle, and affective and marital relationships can be modified. The emotions that accompany this loss process range from shock to denial, from anger to fear, from bitterness to pain, to depression and despair [22].

Embarrassment and shame can be reflected in persecutory experiences such as feeling stigmatized and avoided. Advances in oncology research and new therapeutic strategies with chronicization of oncological disease have made QoL of crucial importance in the patient's survival. This aspect can influence psychological well-being of patient and care adherence [23]. Few studies assessed the psychological impact of disfiguring skin conditions in patients with $\mathrm{mCRC}$ and the extent to which the rash can affect the psychological well-being of patients with skin toxicity had a significant impact on distress [24].

In this study we hypothesized that skin reactions decrease QoL and have a great impact on psycho-social sphere. On these data, we conducted a study to evaluate the skin manifestations induced by cetuximab and QoL of patients, evaluating whether these conditions can lead to impairment of the social sphere, as often happens in patients with alopecia. The aim of this study was to evaluate skin toxicity effects on the QoL and on the social sphere, of patients with colorectal cancer treated with cetuximab. In particular, we also assessed the impact of the rash on body image perception. The primary objective was to investigate the relationship between skin rash and QoL. The second objective was assessing whether QoL affects the psychological perception and whether the treatments affect body shame.

\section{Materials and Methods}

\section{Participants}

All patients with $\mathrm{mCRC}$ who received cetuximab treatment in combination with chemotherapy, from April 2012 to June 2019 , were included in this study. The study was approved by IRB, written informed consent to participate was obtained by all participants. The study was conducted in accordance with the provisions of the Helsinki Declaration and good clinical practice guidelines. The following inclusion criteria were required: patient age $>18$ years; $\mathrm{mCRC}$; at least three cycles of cetuximab treatment plus chemotherapy in disease with expression of EGFR and without RAS gene mutations (wild type: WT); a performance status between 0 and 2 evaluated according to the criteria of the Eastern Cooperative Oncology Group (ECOG). Following a preliminary review to socio-demographic format, subjects who did not have an adequate level of understanding of Italian language and those who had obvious psychiatric disorders (i.e., intellectual disabilities, serious psychotic symptoms) were excluded from the study, which prevented from understanding the research objectives and investigation applications. All participants were informed about the purpose of the study and signed the informed consent prior to evaluation. Those who agreed were asked to complete a protocol containing self-assessment measures with pencil and paper. The completion time was about $20 \mathrm{~min}$. Self-evaluation was carried out at the health facility to ensure data confidentiality. After randomization, patients were monitored for skin rash, adverse events, QoL and perception body image. The reference patient's oncologist evaluated patients for the recording of adverse events according to Common Terminology Criteria for Adverse Events (CTCAEs) version 3.0 [25].

\section{Instruments}

\section{Socio-demographic form}

This module is specially designed to be used during adminis- 
tration, making it useful both for collection socio-demographic data and for understanding disease information. The socio-demographic variables evaluated were: age, sex, place of birth, marital status, education, employment, type of treatment and therapies.

\section{European Organization for Research and Treatment of Can- cer (EORTC-C30)}

The EORTC QLQ-C30 [26-28] is a questionnaire validated by EORTC Group. The questionnaire consists of 30 items on a four-point scale Likert ( $1=$ "no"; $4=$ "very much") that evaluate QoL of cancer patients in the last 7 days. Five items evaluate general function scale (physical, role-playing, emotional, cognitive and social), three items evaluate symptom scales (fatigue, nausea/vomiting and pain), one item evaluates the global health/quality of life scale, and six items evaluate physical questions (breathing, insomnia, anorexia, constipation, diarrhea and economic impact of the disease). All scales and single element sizes vary in score from 0 to 100 . A high score represents a higher level of response. Thus, a high score for a functional scale represents a high/healthy level of functioning, a high score for the overall health status/QoL, but a high score for a symptom physical scale represents a high level of problems.

\section{Body uneasiness test (BUT)}

BUT [29] is self-report psychometric questionnaire for clinical evaluation of discomfort related to body image. The test consists of 71 items with multiple choice responses and is divided into two parts. The first part (BUT a) consists of 34 items whose overall average score identifies the degree of severity related to an individual's body image (Global Severity Index (GSI)). It consists of five areas: weight phobia (WP), body image concerns (BIC), prevention (A), compulsive self-monitoring (CSM), and depersonalization (D). The second part (BUT b) is composed of 37 items that list parts and functions of the body. Gravity is expressed as a scale from 0 to 5 , where 0 corresponds to the absence of problems in that sector and 5 to the maximum gravity. The judgement of gravity is expressed in this case on a scale from 0 to 37 , where 0 corresponds to the absence of the symptom and 37 to the maximum discomfort of the body. BUT test showed good internal consistency with a Cronbach $\alpha$ value of 0.87 .

\section{Statistical analysis}

After checking univariate normality of distributions using asymmetry and kurtosis indices, we tested multivariate normality between variables using Mardia coefficient. The descriptive statistics (mean, standard deviation) were calculated and correlations between the variables were calculated. The reliability of each scale was assessed using Cronbach internal consistency index. The descriptive statistics were used to
Table 1. Socio-Demographic Data $(N=100)$

\begin{tabular}{ll}
\hline Age (range) & $58(35-75)$ \\
Sex & \\
\multicolumn{1}{l}{ Females } & $66(66 \%)$ \\
Males & $34(34 \%)$ \\
ECOG performance status & $14(14 \%)$ \\
0 & $37(37 \%)$ \\
1 & $48(49 \%)$ \\
2 & \\
First line of chemotherapy & $51(51 \%)$ \\
Yes & $49(49 \%)$ \\
No & \\
Civil status & $15(15 \%)$ \\
Single & $76(76 \%)$ \\
Married & $5(5 \%)$ \\
Widowed & $4(4 \%)$ \\
Separated/divorced & \\
Education level & $2(2 \%)$ \\
Primary school & $8(8 \%)$ \\
Secondary school & $53(53 \%)$ \\
High school & $37(37 \%)$ \\
\hline Graduated &
\end{tabular}

ECGO: Eastern Cooperative Oncology Group.

summarize the demographic and study variables. Examining the differences between the participants' average scores and value test (cut-off), $t$-tests of a sample were conducted. The $t$-test was used to compare the mean values of the continuous variables for both BUT and EORTC-C30. A P value of 0.05 was considered statistically significant. The correlation significance between the variables was assessed by Chi-square $\left(\chi^{2}\right)$ test, Pearson's correlation coefficient. All analyses were performed using the statistical software SPSS (Statistical System Statistical Package for Social Sciences) version 25.0 for Mac.

\section{Results}

\section{Socio-demographic characteristics}

A total of 100 patients were enrolled in the study. The normality index verified through univariate indices of asymmetry and kurtosis with 1 acceptance threshold. No variables showed normal violations. The multivariate index of Mardia and kurtosis calculated on variables was 311 , below the critical limit of 399. Patients characteristics with mCRC included in the study are shown in Table 1 . The $66 \%$ of participants were women and $34 \%$ were men. The participants average age was 58 years (range 35 - 75). All participants were Italian. Of the samples, $15 \%$ were single, $76 \%$ were married, $4 \%$ were separated/ di- 
Table 2. Skin Toxicity Using CTCAE $(N=100)$

\begin{tabular}{llllll} 
& Grade 0 & Grade 1 & Grade 2 & Grade 3 & Grade 4 \\
\hline Skin rash & $0(0 \%)$ & $10(10 \%)$ & $35(35 \%)$ & $47(47 \%)$ & $8(8 \%)$ \\
\hline
\end{tabular}

CTCAE: Common Terminology Criteria for Adverse Event.

Table 3. Pearson's Correlation of Skin Rash and QoL in Patients Receiving Cetuximab $(\mathrm{N}=100)$

\begin{tabular}{lll} 
& Skin rash & QoL \\
\hline Skin rash & 1 & $-0.65^{*}$ \\
QoL & $-0.65^{*}$ & 1 \\
\hline
\end{tabular}

${ }^{*} P<0.01$. QoL: quality of life.

vorced and 5\% were widowed. Most samples had a higher level of education $(90 \%)$ (Table 1$)$.

\section{Skin toxicity}

The skin toxicity was assessed at baseline (before starting antineoplastic treatment) and at every subsequent visit, using CTCAE system. The assessment of several aspects of targeted therapy-related adverse events was conducted, using five grades of severity, and the severity is established on the basis of clinical presentation and the reported distress of the patient (such as pruritus and burning). The data showed that none of patients $(0 \%)$ was grade 0,10 patients $(10 \%)$ were grade 1 ,

Table 4. Average Scores and Standard Deviation of Questionnaire EORTC QLQ-C30 (N = 100)

\begin{tabular}{|ll|}
\hline QoL & Average (SD) \\
\hline Functional scale & \\
Physical function & $77.6(16.5)$ \\
\hline Functional role & $65.8(29.4)$ \\
Emotional state & $81.6(23.1)$ \\
\hline Cognitive function & $88.4(26.3)$ \\
Social function & $45.3(21.7)$ \\
Global health/QoL & $55.9(18.5)$ \\
\hline Insomnia & $46.9(18.7)$ \\
\hline Symptom scale & \\
\hline Fatigue & $59.4(26.2)$ \\
Pain & $13.2(25.1)$ \\
\hline Dyspnea & $11.5(21.7)$ \\
\hline Constipation & $19.4(26.1)$ \\
\hline Appetite loss & $28.7(29.2)$ \\
Diarrhea & $22.8(29.7)$ \\
Nausea, vomiting & $41.3(22.6)$ \\
Financial difficulties & $14.7(19.3)$ \\
\hline
\end{tabular}

EORTC QLQ-C30: European Organization for Research and Treatment of Cancer; QoL: quality of life; SD: standard deviation.
Table 5. Average Score and Standard Deviation of BUT Test $(\mathrm{N}=100)$

\begin{tabular}{ll} 
& Average (SD) \\
\hline Weight phobia & $9.7(7.4)$ \\
Concern for the image of the body & $18.2(8.5)$ \\
Avoidance behavior & $16.3(7.8)$ \\
Compulsive control of image & $6.6(16.4)$ \\
Depersonalization & $9.2(11.17)$ \\
\hline
\end{tabular}

BUT: body uneasiness test; SD: standard deviation.

35 patients (35\%) were grade 2,47 patients (47\%) were grade 3 , and eight patients $(8 \%)$ were grade 4 (Table 2$)$. A significant correlation was found between skin rash and overall QoL, with a Pearson correlation coefficient of $-0.65(\mathrm{P}=0.0001)$. The major effects of dermal toxicity on QoL were physical symptoms (e.g., skin itching), while emotional disorders were reported less frequently such as associated to impairment in QoL (Table 3).

\section{QoL}

In general, 78\% of patients interviewed report a good QoL, as shown in Table 4. The data of questionnaire report a greater impact on daily routine, with consequences in relationships and social roles (low scores in the social functioning dimension of EORTC QLQ-C30 scale). The patients express negative feelings such as anxiety, fear and suffering, consistent with an emotional malfunction within the dimension emotional functioning scale of EORTC QLQ-C30. In particular, patients who have performed multiple treatment lines show poor relationships in family and with friends (low scores in the social functioning scales of EORTC QLQ-C30). The 87\% of samples interviewed report insomnia. The most difficult common concerns related to physical well-being were painful, irritating and irritated skin, itching and increased hair growth. A variety of symptoms were seen, such as itching, burning/tingling. These symptoms interfered with sleep, ability to work, their daily activities and their hobbies. In general, QoL decreased during the study course. This significant drop in QoL underlines the continue need looking for interventions that may mitigate this skin-related toxicity in patients receiving EGFR inhibitors.

\section{Psycho-social implications}

The psycho-social dimensions assessed by BUT questionnaire show higher scores for body image with consequence of avoidance behavior of social relationships, leading the subject to isolate themselves (Table 5). Patients reported embarrassment and their skin toxicity interfered with their ability to show affection. Most patients have felt a sense of loneliness, with difficulty leaving home and delegated their family member to communicate with friends. Of patients, 52\% responded that they had avoided going out, experienced concern, frustration 
and depression, due to their dermatological rash. Data suggest that skin toxicity associated with EGFR inhibitors had a profound impact on physical function, emotional and social of the patient also interfering with treatment compliance. Fear, frustration and depression were related to their skin symptoms and concerned about the scarring from skin toxicity. High score was found in social discomfort dimension with isolation behaviors also for work, related to concerns about how others would react.

\section{Discussion}

EGFR antibodies, associated with chemotherapy, prolong survival in mCRC. However, often in patients, it develops severe skin toxicity. This occurs in about $80 \%$ of treated patients, negatively affecting their QoL and their resistance to disease $[15,16]$. The psycho-educational approach, with correct information on the side effects and their possible treatment, could play a decisive role in therapeutic compliance and psychological well-being of patient [30]. In this study, rash had a negative impact on QoL and body perception. Social relationships were avoided through embarrassment, unease and body shaming. The results confirmed the correlation between skin manifestations and compromised QoL. Therefore, QoL and perception of body image influence the acceptance of the diagnosis, better therapeutic compliance, disease adaptation and consequently a greater acceptance of side effects [16-31]. From data of our study, patients did not believe skin toxicity as a priority during their treatment, but their management caused distress. Both during and after treatment, the attention to the side effects of chemotherapy, in particular management of the skin rash, is a priority in $\mathrm{mCRC}$ patients. The main objective of this research was therefore to evaluate psychological well-being, implement effective interventions in order to reduce physical and psychological discomfort and motivate patients to tolerate symptoms because the toxicity is often index to treatment response. In fact, when the followup shows a good response to treatment, the patient accepts the skin toxicity with less distress. The neoplastic disease is characterized as a complex pathology which requires a multidisciplinary and integrated approach to the patient that also involves the psycho-oncologist, which is able to evaluate its different aspects and its different implications, not only from a physical but also psychological point of view [32-34]. The psycho-oncologist is of fundamental importance exactly in patient's subjective perception and coping strategies to face up the disease. The psychological intervention involves taking care of the patient through weekly interviews and the administration of psychometric tools, promoting adaptive coping strategies that allow for patient not to break down and surrender to the first symptoms of rash. Although significant QoL alteration has been observed in patients receiving EGFR inhibitors, skin rash must be addressed in supportive interventions in routine management before this hinders treatment. Psycho-oncological work should focus on minimizing distress and developing active coping strategies, motivating the patient not to stop treatment for the effectiveness of the drug.

\section{Acknowledgments}

None to declare.

\section{Financial Disclosure}

None to declare.

\section{Conflict of Interest}

None to declare.

\section{Informed Consent}

All patients signed informed consent.

\section{Author Contributions}

RDL, GLC, RP, OM, MSE: data collection; RA, GC: conceptualization, manuscript revision. SISF, AA, AS, ADA: figures preparation writing original draft preparation. All authors reviewed the manuscript.

\section{Data Availability}

The data supporting the findings of this study are available from the corresponding author upon reasonable request.

\section{References}

1. Jemal A, Murray T, Ward E, Samuels A, Tiwari RC, Ghafoor A, Feuer EJ, et al. Cancer statistics, 2005. CA Cancer J Clin. 2005;55(1):10-30.

2. Cicero G, De Luca R, Dieli F. Efficacy and safety of the oral multikinase regorafenib in metastatic colorectal cancer. Oncology. 2017;93(6):354-358.

3. Cicero G, Lo Re G, R DEL, Vernuccio F, Picone D, Midiri $\mathrm{M}$, Lagalla R. Role of densitometric criteria in evaluation of effectiveness of antiangiogenic therapies in metastatic colorectal cancer: an Italian clinical experience. Anticancer Res. 2017;37(9):5187-5192.

4. Hsu HT, Wu LM, Lin PC, Juan CH, Huang YY, Chou PL, Chen JL. Emotional distress and quality of life during folinic acid, fluorouracil, and oxaliplatin in colorectal cancer patients with and without chemotherapy-induced peripheral neuropathy: A cross-sectional study. Medicine (Baltimore). 2020;99(6):e19029.

5. Fernandez-Martos C, Pericay C, Aparicio J, Salud A, Safont M, Massuti B, Vera R, et al. Phase II, randomized study of concomitant chemoradiotherapy followed by surgery and adjuvant capecitabine plus oxaliplatin (CA- 
POX) compared with induction CAPOX followed by concomitant chemoradiotherapy and surgery in magnetic resonance imaging-defined, locally advanced rectal cancer: Grupo cancer de recto 3 study. J Clin Oncol. 2010;28(5):859-865.

6. Cunningham D, Humblet Y, Siena S, Khayat D, Bleiberg H, Santoro A, Bets D, et al. Cetuximab monotherapy and cetuximab plus irinotecan in irinotecan-refractory metastatic colorectal cancer. N Engl J Med. 2004;351(4):337345.

7. Labianca R, La Verde N, Garassino MC. Development and clinical indications of cetuximab. Int J Biol Markers. 2007;22(1 Suppl 4):S40-46.

8. Marventano S, Forjaz M, Grosso G, Mistretta A, Giorgianni G, Platania A, Gangi S, et al. Health related quality of life in colorectal cancer patients: state of the art. BMC Surg. 2013;13(Suppl 2):S15.

9. Sultan S, Fisher DA, Voils CI, Kinney AY, Sandler RS, Provenzale D. Impact of functional support on healthrelated quality of life in patients with colorectal cancer. Cancer. 2004;101(12):2737-2743.

10. Au HJ, Karapetis CS, O'Callaghan CJ, Tu D, Moore MJ, Zalcberg JR, Kennecke H, et al. Health-related quality of life in patients with advanced colorectal cancer treated with cetuximab: overall and KRAS-specific results of the NCIC CTG and AGITG CO.17 Trial. J Clin Oncol. 2009;27(11):1822-1828.

11. Li T, Perez-Soler R. Skin toxicities associated with epidermal growth factor receptor inhibitors. Target Oncol. 2009;4(2):107-119.

12. Boone SL, Rademaker A, Liu D, Pfeiffer C, Mauro DJ, Lacouture ME. Impact and management of skin toxicity associated with anti-epidermal growth factor receptor therapy: survey results. Oncology. 2007;72(3-4):152-159.

13. Sipples R. Common side effects of anti-EGFR therapy: acneform rash. Semin Oncol Nurs. 2006;22(1 Suppl 1):28-34.

14. Melosky B, Burkes R, Rayson D, Alcindor T, Shear N, Lacouture M. Management of skin rash during EGFR-targeted monoclonal antibody treatment for gastrointestinal malignancies: Canadian recommendations. Curr Oncol. 2009;16(1):16-26.

15. Matzka M, Stohr D, Colditz A, Kock-Hodi S, Koller M, Mayer H. Skin toxicities and unmet supportive care needs of patients with cancer undergoing EGFR-inhibitor therapy. Pflege. 2017;30(1):9-17.

16. Jatoi A, Rowland K, Sloan JA, Gross HM, Fishkin PA, Kahanic SP, Novotny PJ, et al. Tetracycline to prevent epidermal growth factor receptor inhibitor-induced skin rashes: results of a placebo-controlled trial from the North Central Cancer Treatment Group (N03CB). Cancer. 2008;113(4):847-853.

17. Unger K, Niehammer U, Hahn A, Goerdt S, Schumann M, Thum S, Schepp W. Treatment of metastatic colorectal cancer with cetuximab: influence on the quality of life. Z Gastroenterol. 2013;51(8):733-739.

18. LoRusso P. Toward evidence-based management of the dermatologic effects of EGFR inhibitors. Oncology (Williston Park). 2009;23(2):186-194.
19. De Luca R, Lo Coco G, Ardizzone A, et al. Psychological distress and quality of life in ostomy patients with colorectal cancer: a systematic review of the literature. Acta Medica Mediterranea. 2018;34:1357.

20. Lemieux J, Maunsell E, Provencher L. Chemotherapyinduced alopecia and effects on quality of life among women with breast cancer: a literature review. Psychooncology. 2008;17(4):317-328.

21. Dunn J, Lynch B, Aitken J, Leggett B, Pakenham K, Newman B. Quality of life and colorectal cancer: a review. Aust N Z J Public Health. 2003;27(1):41-53.

22. Lacouture ME, Mitchell EP, Piperdi B, Pillai MV, Shearer $\mathrm{H}$, Iannotti N, Xu F, et al. Skin toxicity evaluation protocol with panitumumab (STEPP), a phase II, open-label, randomized trial evaluating the impact of a pre-Emptive Skin treatment regimen on skin toxicities and quality of life in patients with metastatic colorectal cancer. J Clin Oncol. 2010;28(8):1351-1357.

23. Schover LR. The impact of breast cancer on sexuality, body image, and intimate relationships. CA Cancer J Clin. 1991;41(2):112-120.

24. Lethborg C, Aranda S, Cox S, Kissane D. To what extent does meaning mediate adaptation to cancer? The relationship between physical suffering, meaning in life, and connection to others in adjustment to cancer. Palliat Support Care. 2007;5(4):377-388.

25. Romito F, Giuliani F, Cormio C, Tulipani C, Mattioli V, Colucci G. Psychological effects of cetuximab-induced cutaneous rash in advanced colorectal cancer patients. Support Care Cancer. 2010;18(3):329-334.

26. Trotti A, Colevas AD, Setser A, Rusch V, Jaques D, Budach V, Langer C, et al. CTCAE v3.0: development of a comprehensive grading system for the adverse effects of cancer treatment. Semin Radiat Oncol. 2003;13(3):176181.

27. Snyder CF, Blackford AL, Okuyama T, Akechi T, Yamashita H, Toyama T, Carducci MA, et al. Using the EORTC-QLQ-C30 in clinical practice for patient management: identifying scores requiring a clinician's attention. Qual Life Res. 2013;22(10):2685-2691.

28. Sprangers MA, Cull A, Bjordal K, Groenvold M, Aaronson NK. The European Organization for research and treatment of cancer. Approach to quality of life assessment: guidelines for developing questionnaire modules. EORTC Study Group on Quality of Life. Qual Life Res. 1993;2(4):287-295.

29. Cuzzolaro M, Vetrone G, Marano G, et al. Body uneasiness test BUT, in Conti L., a cura di: Repertorio delle scale di valutazione in psichiatria. SEE. 2000;3(1)759761.

30. Teker F, Demirag G, Erdem D, et al. Quality of life in colorectal cancer patients during chemotherapy inthe era of monoclonal antibody therapies. J Buon. 2015;20(2):4351.

31. Lynne I, Wagner ME. La couture, dermatologic toxicities associated with EGFR Inhibitors. Palliative and Supportive Care. 2007;21(5):11.

32. Bruheim K, Guren MG, Dahl AA, Skovlund E, Balteskard L, Carlsen E, Fossa SD, et al. Sexual function in males af- 
ter radiotherapy for rectal cancer. Int $\mathrm{J}$ Radiat Oncol Biol Phys. 2010;76(4):1012-1017.

33. Wagner LI, Lacouture ME. Dermatologic toxicities associated with EGFR inhibitors: the clinical psychologist's perspective. Impact on health-related quality of life and implications for clinical management of psychological sequelae. Oncology (Williston Park). 2007;21(11 Suppl 5):34-36.

34. Cicero G, De Luca R, Dieli F. Progression-free survival as a surrogate endpoint of overall survival in patients with metastatic colorectal cancer. Onco Targets Ther. 2018;11:3059-3063. 